in group 2 reported some adverse events versus only $27.8 \%$ in elderly patients.

Conclusion In Croatia, from February 2017 we have opportunity to treat patients with epithelial ovarian, fallopian tube, or primary peritoneal cancer with bevacizumab in the first-line and second-line settings. Our experience in treating patients with bevacizumab shows good results with acceptable toxicity and our findings suggest that its use in the elderly population should be considered as safe and manageable.

Disclosures The authors have declared no conflicts of interest.

\section{MALIGNANT OVARIAN TUMORS IN PREGNANCY: A CASE SERIES}

Helena Azimi. Mashhad University of Medical Sciences

\subsection{6/ijgc-2020-ESGO.142}

Introduction/Background Adnexal masses are commonly detected during routine fetal ultrasound screening. Nonetheless, since malignant adnexal tumors rarely occur in pregnancy, limited data is available regarding the management of this condition. Herein, we describe a series of ovarian cancer cases diagnosed and managed during pregnancy.

Methodology This case series describes 22 pregnant patients with ovarian cancer who were referred to the gynecology oncology department of an academic hospital within 6 years. Demographic and clinical characteristics of cases were gathered in checklists. Surgical staging of the tumors as well as diseasefree survival (DFS) and overall survival (OS) were determined in all patients.

Results The pathologic subtype in $45.4 \%$ of the patients was epithelial. In another $45.4 \%$, the subtype was germ cell, and the remaining $9.1 \%$ had sex-cord tumors. In epithelial tumors, the most common subtype was serous adenocarcinoma (60\%). Most of the patients had a palpable mass during physical examination $(72.7 \%)$ or an adnexal mass in ultrasonography (95.4\%). We performed fertility-preserving surgery on 14 patients (63.6\%) and $13(59 \%)$ patients received chemotherapy. The recurrence rate was $22.7 \%$ and DFS and OS were $56 \%$ and $82 \%$, respectively in a 6-year follow-up.

Conclusion Ovarian malignancy is a rare event during pregnancy and its management requires an experienced multidisciplinary approach. Further studies with larger sample sizes are required to provide more insight into the management of ovarian cancer throughout pregnancy.

Disclosures None.

\section{LIQUID BIOPSY FOR DIAGNOSING OVARIAN CANCER- QUANTIFICATION OF CELL-FREE DNA AND P53 MUTATIONAL ANALYSIS}

\footnotetext{
${ }^{1}$ Shalini Rajaram, ${ }^{2}$ Aanchal Verma, ${ }^{3}$ Rajarshi Kar, ${ }^{4}$ Vinod Kumar Arora, ${ }^{5}$ Bindiya Gupta, ${ }^{6}$ Sandhya Jain. ' All India Institute of Medical Sciences - Rishikesh; Division of Gynecologic Oncology; ${ }^{2}$ Guru Teg Bahadur Hospital and University College of Medical Sciences; Obstetrics and Gynecology; ${ }^{3}$ Guru Tegh Bahadur Hospital and University College of Medical Sciences; Department of Biochemistry; ${ }^{4}$ Guru Teg Bahadur Hospital and University College of Medical Sciences; Department of Pathology; ${ }^{5} 1405$ Millenia Heights Ramprastha Greens Sector 7 Vaishali; Guru Teg Bahadur Hospital and University College of Medical Sciences; Department of Obstetrics and Gynecology; ${ }^{6}$ Guru Teg Bahadur Hospital and University College of Medical Sciences; Obstetrics and Gynecology
}

Introduction/Background Circulating tumor DNA found in women with malignancy, enters plasma due to lysis of cells at the interface between the primary tumor and the circulation. The primary objective of this study was to isolate and quantify cell-free DNA (cfDNA) from peripheral blood, analyze p53 mutations and correlate with tumor burden in epithelial ovarian malignancy. Secondary objective was to study the degree of agreement between cfDNA p53 mutations and tissue p53 immunohistochemistry.

Methodology This prospective case-control study was carried out over 18 months from November 2018 to April 2020 at a tertiary teaching institution. Considering the exploratory nature of the study, study group $(n=20)$ comprised women with epithelial ovarian malignancy. Control groups were women with borderline tumors $(n=10)$ and benign epithelial ovarian tumors $(n=10) .58$ women who were treatment naïve and admitted for surgery entered the study but only those with a final histopathology of epithelial ovarian tumor (malignant, borderline and benign) were included. Peritoneal carcinomatosis index (PCI), surgical complexity score and cytoreductive score was calculated in women undergoing primary cytoreduction.

Plasma samples for cfDNA was collected just before surgery and stored at $-20 \mathrm{oC}$. cfDNA was extracted from plasma serum using a DNA isolation kit and quantified with Nanodrop Spectrophotometer. ARMS PCR was used to detect a point mutation in Exon 8, codon 239 of p53 using primer pairs. p53 immunostaining was performed on tissue samples using monoclonal antibody directed against p53. Statistical analysis was done using SPSS version 21.

Results In women with malignant ovarian cancer isolated cfDNA was highest $(1330 \mathrm{ng} / \mathrm{mL})$ in comparison to those with benign or borderline ovarian tumors $(748.5 \mathrm{ng} / \mathrm{mL}$ and $448.5 \mathrm{ng} / \mathrm{mL}$, respectively) reaching statistical significance, $\mathrm{p}=0.023$. Quantity of cfDNA also correlated well with the histopathological grade of the tumor and stage of the disease, $\mathrm{p}<0.05$.

Analysis of cfDNA p53 mutation in exon 8 showed that $55 \%$ of the women diagnosed with malignant ovarian tumor harboured this mutation $(p=0.043)$. Correlation of tissue p53 with cfDNA p53 mutation was statistically significant, $p=0.007$. All women with malignant ovarian tumor in whom cfDNA p53 mutation was present at codon 239 of exon 8 stained positive for tissue p53 mutation.

Conclusion cfDNA p53 mutation in exon 8 was detected at higher frequency in women with malignant epithelial ovarian cancer. Significant correlation was seen between tissue p53 and cfDNA p53 mutation suggesting that mutational analysis of cfDNA could act as biomarker for the diagnosis of ovarian tumors.

Disclosures Shalini Rajaram - No conflict of interest

Aanchal Verma - No conflict of interest

Rajarshi Kar - No conflict of interest

Vinod Arora - No conflict of interest

Bindiya Gupta - No conflict of interest

Sandhya Jain - No conflict of interest 